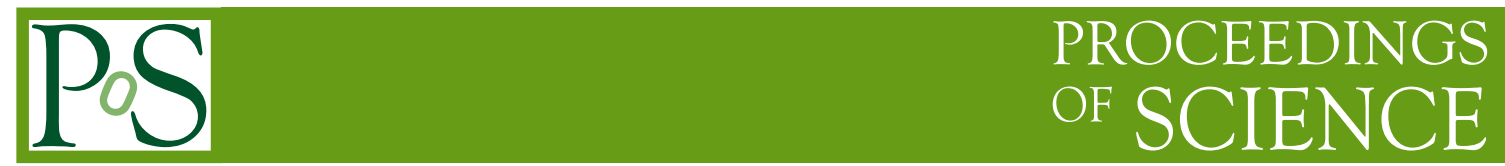

\title{
Charmed hadron decays at BESIII
}

\section{De Mori F.*广}

Università degli studi di Torino and INFN sez. Torino

E-mail: demori@to.infn.it

The BESIII Experiment at the Beijing Electron Positron Collider (BEPCII) has accumulated the world's largest samples of $e^{+} e^{-}$collisions in the tau-charm region. Based on the samples taken at $\Psi(3770)$ peak and at the $\Lambda_{c}^{+} \Lambda_{c}^{-}$mass threshold of $4.599 \mathrm{GeV}$, we can study the charmed hadron decays in a uniquely clean environment. In this report, I will review few of the recent results on the $\mathrm{D}$ and $\Lambda_{c}$ decays.

XXVII International Symposium on Lepton Photon Interactions at High Energies 17-22 August 2015

Ljubljana, Slovenia

* Speaker.

${ }^{\dagger}$ on behalf of BESIII collaboration 
The analyses presented in this report are based on two data samples collected by the BESIII detector [1] installed at the double-ring BEPCII collider (IHEP, Beijng, P.R.C). The first one, used for the D-mesons decay analyses, is the largest $e^{+} e$ annihilation sample in the world to date, $2.92 f b^{1}$, at the $3.773 \mathrm{GeV}$ center-of-mass energy, where $e^{+} e \rightarrow \Psi(3770) \rightarrow D \bar{D}$ is mainly produced. The second sample, that is used for the $\Lambda_{c}^{+}$decays, is the unique and largest, $0.5 f b^{1}$, at the centerof-mass energy of $4.599 \mathrm{GeV}$ which is the $\Lambda_{c}^{+} \Lambda_{c}^{-}$mass threshold. In both cases the "tagging" technique can be exploited because both the $\mathrm{D}$ and the $\Lambda_{c}^{+}$are produced in pairs. As an example, once a D-meson (tagged) hadronic decay has been fully reconstructed in an event, the existence of the other D decay (signal) in the same event is guaranteed. In the following charge conjugation is implied.

\section{D-mesons purely leptonic decay}

In the Standard Model (SM) the rate of the $D^{+} \rightarrow \mu^{+} v_{\mu}$ decay, via a virtual $W$-boson, can be parametrized by the $D^{+}$decay constant $f_{D^{+}}$as $\Gamma\left(D^{+} \rightarrow \mu^{+} v_{\mu}\right)=\frac{G_{F}^{2}}{8 \pi} f_{D^{+}}^{2}\left|V_{c d}\right|^{2} m_{l}^{2} m_{D^{+}}\left(1-\frac{m_{l}^{2}}{m_{D^{+}}^{2}}\right)^{2}$ where $G_{F}$ is the Fermi coupling constant, $\left|V_{c d}\right|$ is the quark mixing matrix element, $m_{l}$ and $m_{D}^{+}$are the lepton and $D^{+}$masses. The measurement of the branching ratio allows to determine the $f_{D^{+}}\left|V_{c d}\right|$ product. To investigate this channel a singly tagged $D$ meson is reconstructed by 9 hadronic decays. The results of fitting to the beam-energy-constrained mass $\left(M_{B C}\right)$ spectra are shown in figure 1 on the left. The total yield of singly tagged $D$ mesons is $(170.31 \pm 0.34) \times 10^{4}$. The $\mu^{+}$is searched in the remaining tracks. On the right of figure 1 the squared missing mass distribution for the selected events is shown with the background simulations. From the 409.0 $\pm 21.2 \pm 2.3$ signal events, a branching fraction of $(3.71 \pm 0.19$ stat. \pm 0.06 sys. $) \times 10^{-4}$ is found. Using the measured $B\left(D^{+} \rightarrow \mu^{+} v_{\mu}\right)$ and the quark mixing matrix element $\left|V_{c d}\right|$ from SM constraint fit [4] the $\mathrm{D}^{+}$ decay constant $f^{D^{+}}$value is determined to be $(203.2 \pm 5.3$ stat. \pm 1.8 sys. $\mathrm{MeV})$. The measured $B$ and $f^{D^{+}}$are consistent within errors with previous results [3], but with the best precision.

Until now the quark mixing matrix element $I V_{c d} \mid$ has been measured using the D-meson semileptonic decays or measuring charm production cross section in $v v$ interactions reaching the best
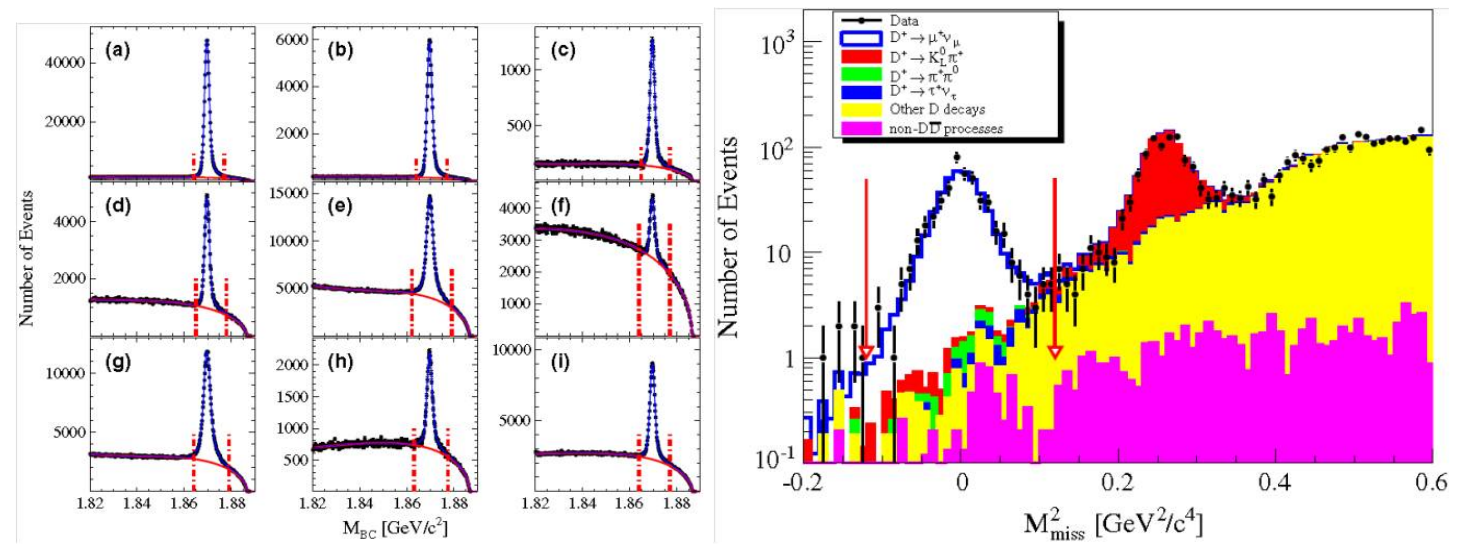

Figure 1: Fit results of $M_{B C}$ spectra from singly tagged $D^{-}$candidates on the left and squared missing mass distribution for the signal candidates on the right. In all the plots the red vertical lines identify the signal region. 
precision of 4.8\% [4]. The high precision of recent Lattice QCD (LQCD) calculation of $f^{D^{+}}$[5] combined with the measured $\mathscr{B}\left(D^{+} \rightarrow \mu^{+} v_{\mu}\right)$, allows to determine $\left|V_{c d}\right|$ with a result of $(0.2210 \pm$ 0.058 stat. \pm 0.047 sys. $)$, reaching the best precision in the world. For more details about this analysis refer to [2].

\section{2. $D^{0}$-mesons semi-leptonic decays}

The differential decay rate of $D^{0} \rightarrow K^{-}\left(\pi^{-}\right) e^{+} v$ can be written as $\frac{d \Gamma}{d q^{2}}=\frac{G_{F}^{2}\left|V_{c s(d)}\right|^{2}}{24 \pi^{3}} p^{3}\left|f_{+}^{K(\pi)}\left(q^{2}\right)\right|^{2}$ where $\mathrm{p}$ is the kaon (pion) momentum in the $D^{0}$ rest frame, while $f_{+}^{K(\pi)}\left(q^{2}\right)$ is the form factor of hadronic weak current and $\mathrm{q}$ is the four momentum transfer $q=P_{D}-P_{K(\pi)}$. In this analysis the singly tagged $\overline{D^{0}}$ mesons are reconstructed using 5 hadronic decays. Fitting $M_{B C}$ spectra $(279.33 \pm$ $0.37) \times 10^{4}$ singly tagged $\overline{D^{0}}$ mesons are found. The signal candidates are searched by reconstructing two opposite charged tracks left in the event and $U_{\text {miss }}=E_{\text {miss }}-p_{\text {miss }}$ is used to select signal events, obtaining $70727 \pm 278$ and $6297 \pm 87$ signal events for the $\mathrm{K}$ and $\pi$ channel, respectively. The measured branching fraction are $\mathscr{B}\left(D^{0} \rightarrow K^{-} e^{+} v_{e}\right)=(3.505 \pm 0.014$ stat. \pm 0.033 sys. $) \%$ and $\mathscr{B}\left(D^{0} \rightarrow \pi^{-} e^{+} v_{e}\right)=(0.295 \pm 0.004$ stat. \pm 0.003 sys. $) \%$, consistent within errors with previous measurements [7], but with the best precision. Fitting the partial widths with different parametrizations the values of $\left|f_{+}^{K(\pi)}(0)\right|\left|V_{c s(d)}\right|$ are extracted. Using the precise $\left|V_{c s(d)}\right|$ from the SM constraint fit [4] we can obtain the form factors $\left|f_{+}^{K(\pi)}(0)\right|$ to check LQCD calculations whose precision is largely increasing. In figure 2 the comparison of the measured form factors with the LQCD calculations [8] is shown. For more details about this analysis refer to [6].
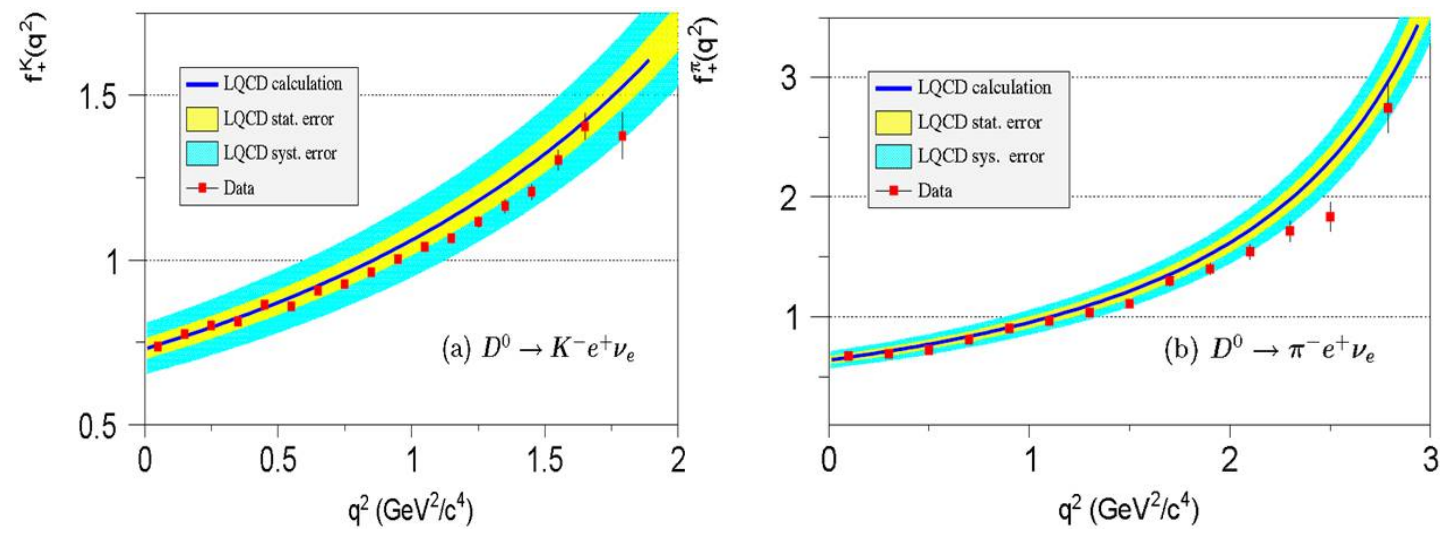

Figure 2: Comparison of the measured form factor, represented by the squares with error bars, as a function of $q^{2}$ with the LQCD calculation [8] for $D^{0} \rightarrow \pi e^{+} v_{e}$ on the left and $D^{0} \rightarrow K e^{+} v_{e}$ on the right. The couloured bands represent the error bands of the theoretical calculation, that is the solid line.

\section{3. $\Lambda_{c}^{+}$hadronic decays}

Most of the $\Lambda_{c}^{+}$decay rates have been experimentally determine referred to the channel $\Lambda_{c}^{+} \rightarrow p K^{-} \pi^{+}$. The measured branching fraction (BF) of that channel has suffered large uncertainty due to inclusive model assumption until the recent Belle [10] measurement, that improved by a factor of 5 the precision. Cabibbo-favored (CF) hadronic BFs have still poor 
precisions. Based on our unique data sample at $4.599 \mathrm{GeV}$, we measured simultaneously the absolute BFs of $12 \mathrm{CF}$ hadronic decays [9] for the first time with a model-independent method at $\Lambda_{c}^{+} \Lambda_{c}^{-}$production threshold by means of the double-tag technique. The results are reported in table 1 with the PDG [4] values for comparison. For BESIII results the first errors are statistical, the second are systematic which combined errors from external input BFs of the intermediate decays. Our result of $\mathscr{B}\left(\Lambda_{c} \rightarrow p K \pi^{+}\right)$is consistent with the PDG value but lower with a significance of about $2 \sigma$ than the $\mathscr{B}=\left(6.84 \pm 0.24_{-0.27}^{+0.21}\right) \%$ measured by Belle[10]. For the other modes the precision is incresead from 3 to 6 times.

\begin{tabular}{|c|c|c|c|c|c|}
\hline Mode & $\mathscr{B}(\%)$ & PDG $(\%)$ & Mode & $\mathscr{B}(\%)$ & PDG $(\%)$ \\
\hline$p K_{S}^{0}$ & $1.52 \pm 0.08 \pm 0.03$ & $1.15 \pm 0.30$ & $\mathrm{p} K \pi^{+}$ & $5.84 \pm 0.27 \pm 0.23$ & $5.0 \pm 1.3$ \\
\hline Mode & $\mathscr{B}(\%)$ & PDG $(\%)$ & Mode & $\mathscr{B}(\%)$ & PDG $(\%)$ \\
\hline$p K_{S}^{0} \pi^{0}$ & $1.87 \pm 0.13 \pm 0.05$ & $1.65 \pm 0.50$ & $p K_{S}^{0} \pi^{+} \pi$ & $1.53 \pm 0.11 \pm 0.09$ & $1.30 \pm 0.35$ \\
\hline Mode & $\mathscr{B}(\%)$ & PDG $(\%)$ & Mode & $\mathscr{B}(\%)$ & PDG $(\%)$ \\
\hline $\mathrm{p} K \pi^{+} \pi^{0}$ & $4.53 \pm 0.23 \pm 0.30$ & $3.4 \pm 1.0$ & $\Lambda \pi^{+}$ & $1.24 \pm 0.07 \pm 0.03$ & $1.07 \pm 0.28$ \\
\hline Mode & $\mathscr{B}(\%)$ & PDG $(\%)$ & Mode & $\mathscr{B}(\%)$ & PDG (\%) \\
\hline$\Lambda \pi^{+} \pi^{0}$ & $7.01 \pm 0.37 \pm 0.19$ & $3.6 \pm 1.3$ & $\Lambda \pi^{+} \pi \pi^{+}$ & $3.81 \pm 0.24 \pm 0.18$ & $2.6 \pm 0.7$ \\
\hline Mode & $\mathscr{B}(\%)$ & PDG $(\%)$ & Mode & $\mathscr{B}(\%)$ & PDG $(\%)$ \\
\hline$\Sigma^{0} \pi^{+}$ & $1.27 \pm 0.08 \pm 0.03$ & $1.05 \pm 0.28$ & $\Sigma^{+} \pi^{0}$ & $1.18 \pm 0.10 \pm 0.03$ & $1.00 \pm 0.34$ \\
\hline Mode & $\mathscr{B}(\%)$ & PDG $(\%)$ & Mode & $\mathscr{B}(\%)$ & PDG $(\%)$ \\
\hline$\Sigma^{+} \pi^{+} \pi$ & $4.25 \pm 0.24 \pm 0.20$ & $3.6 \pm 1.0$ & $\Sigma^{+} \omega$ & $1.56 \pm 0.20 \pm 0.07$ & $2.7 \pm 1.0$ \\
\hline \hline
\end{tabular}

Table 1: In this table the branching fractions obtained in the BESIII analysis are compared to the PDG values [4].

\section{References}

[1] M. Ablikim et al. (BESIII Collaboration), Nucl. Instrum. Methods Phys. Res. A 614, 345 (2010).

[2] M. Ablikim, et al. (BESIII Collaboration), Phys. Rev. D 89, 051104(R) (2014).

[3] J. Z. Bai, et al. (BES Collaboration), Phys. Lett. B 429, 188 (1998);M. Ablikim, et al. (BES Collaboration), Phys. Lett. B610, 173 (2005);B. I. Eisenstein et al. (CLEO Collaboration), Phys. Rev. D 78, 052003 (2008).

[4] K.A. Olive et al. (Particle Data Group), Chin. Phys. C 38, 090001 (2014)

[5] E. Follana et al. (HPQCD and UKQCD Collaborations),Phys. Rev. Lett. 100, 062002 (2008).

[6] M. Ablikim, et al. (BESIII Collaboration), Phys. Rev. D 92,072012(2015).

[7] M. Ablikim, et al. (BES Collaboration), Phys. Lett. B 597, 39 (2005);D. Besson, et al. (CLEO Collaboration), Phys. Rev. D 80, 032005 (2009);L. Widhalm, et al. (Belle Collaboration), Phys. Rev. Lett. 97, 061804 (2006);B. Aubert et al. (BABAR Collaboration), Phys. Rev. D 76, 052005 (2007);J. P. Lees et al. (BABAR Collaboration), Phys. Rev. D 91, 052022 (2015).

[8] C. Aubin et al.,Phys. Rev. Lett.94, 011601(2005).

[9] M. Ablikim, et al. (BESIII Collaboration), submitted to PRL.

[10] A. Zupanc et al (Belle Collaboration), Phys. Rev. Lett. 113, 042002 (2014). 